\title{
Scrape-off layer power fall-off length from turbulence simulations of ASDEX Upgrade L-mode \\ Paper
}

Olsen, Jeppe; Nielsen, Anders Henry; Rasmussen, Jens Juul; Madsen, Jens; Eich, Thomas; Sieglin, Bernhard; Naulin, Volker

Published in:

Plasma Physics and Controlled Fusion

Link to article, DOI:

10.1088/1361-6587/aace8b

Publication date:

2018

Document Version

Peer reviewed version

Link back to DTU Orbit

Citation (APA):

Olsen, J., Nielsen, A. H., Rasmussen, J. J., Madsen, J., Eich, T., Sieglin, B., \& Naulin, V. (2018). Scrape-off layer power fall-off length from turbulence simulations of ASDEX Upgrade L-mode: Paper. Plasma Physics and Controlled Fusion, 60(8), [085018]. https://doi.org/10.1088/1361-6587/aace8b

\section{General rights}

Copyright and moral rights for the publications made accessible in the public portal are retained by the authors and/or other copyright owners and it is a condition of accessing publications that users recognise and abide by the legal requirements associated with these rights.

- Users may download and print one copy of any publication from the public portal for the purpose of private study or research.

- You may not further distribute the material or use it for any profit-making activity or commercial gain

- You may freely distribute the URL identifying the publication in the public portal 


\section{Scrape-off layer power fall-off length from turbulence simulations of ASDEX Upgrade L-mode}

\section{Jeppe Olsen}

PPFE, Department of Physics, Technical University of Denmark, Building 309, 2800

Kgs. Lyngby

E-mail: jmbols@fysik.dtu.dk

\section{Anders Henry Nielsen}

PPFE, Department of Physics, Technical University of Denmark, Building 309, 2800 Kgs. Lyngby

E-mail: ahnie@fysik.dtu.dk

\section{Jens Juul Rasmussen}

PPFE, Department of Physics, Technical University of Denmark, Building 309, 2800 Kgs. Lyngby

E-mail: jjra@fysik.dtu.dk

\section{Jens Madsen}

PPFE, Department of Physics, Technical University of Denmark, Building 309, 2800 Kgs. Lyngby

E-mail: jmad@fysik.dtu.dk

\section{Thomas Eich}

Max-Planck Institute for Plasma Physics, Boltzmannstrasse 2, Garching, Germany

E-mail: thomas.eich@ipp.mpg.de

\section{Bernhard Sieglin}

Max-Planck Institute for Plasma Physics, Boltzmannstrasse 2, Garching, Germany

E-mail: bernhard.sieglin@ipp.mpg.de

\section{Volker Naulin}

PPFE, Department of Physics, Technical University of Denmark, Building 309, 2800 Kgs. Lyngby

E-mail: vona@fysik.dtu.dk 


\begin{abstract}
The scrape-off layer power fall-off length, $\lambda_{q}$, for parameters relevant to ASDEX Upgrade (AUG) L-mode discharges is examined by means of numerical simulations. The simulation data is acquired using synthetic probe data from turbulence simulations, which gives a high temporal resolution on the full density and pressure fields, required for an accurate evaluation of $\lambda_{q}$ due to the strongly intermittent signals in the scrape-off layer (SOL). Electron conduction is found to dominate the parallel heat flux close to the separatrix, while ion convection dominates in the far SOL. Good agreement is found with the experimental scaling for AUG Lmode [Sieglin et al., Plasma Phys. Contr. Fusion 2016;58(5):055015], where $\lambda_{q}$ is found to scale almost linearly with the safety factor, $q$, and to be weakly dependent on the power across the last-closed flux surface (LCFS), $P$. However, $P$ depends on a wide range of parameters. In this paper we trace this dependence and the resulting fit of $\lambda_{q}$ reveals a scaling proportional to the inverse square root of the electron temperature at the LCFS, $T_{e, L C F S}^{-1 / 2}$, and a linear dependence on $q$.
\end{abstract}

Keywords: Scrape-off layer power fall-off length, scrape-off layer transport, scrape-off layer parallel heat flux

Submitted to: Plasma Phys. Control. Fusion

\title{
1. Introduction
}

In magnetic confinement fusion devices most of the power across the last-closed flux surface (LCFS) is transported towards the divertor along magnetic field lines in a narrow region close to the separatrix [1]. The width of this channel is denoted as $\lambda_{q}$ and it is crucial to know this parameter when determining the divertor peak heat load, which for ITER steady state operation needs to be kept below $10 \mathrm{MWm}^{-2}$ due to material constraints. To estimate $\lambda_{q}$ for ITER, several empirical scaling laws have been derived based on engineering parameters and extrapolated to ITER relevant parameters, both for limited plasmas [2-4] and for divertor plasmas [5-8].

The different scaling laws, however, do not all agree and it is not yet clear how much individual heat flux channels contribute to $\lambda_{q}$. In the experimental scaling laws, $\lambda_{q}$ is either determined at the outer mid-plane using reciprocating Langmuir probes (as in [3]) or at the divertor using infra-red spectrometry and then mapping it to the outboard mid-plane (as in [1]). Neither of these methods provide insight into the individual contributions from electrons and ions to $\lambda_{q}$, and both are subject to large uncertainties when estimating $\lambda_{q}$ and the corresponding parameters, e.g., when determining the position of the LCFS. For larger machines like AUG, it has been shown that the width of the parallel heat flux channel, $\lambda_{q}$, and the width of the parallel electron temperature channel, $\lambda_{T e}$, even when in L-Mode conditions, are well correlated by Spitzer-Härm conduction and hence $\lambda_{q} / \lambda_{T e}=2 / 7$ is found $[9,10]$. This correlation, however, is doubtful for smaller machines like TCV and Compass, so an experimental 
multi-machine scaling for the power width in L-Mode conditions is difficult to establish and prone to artefacts; therefore this paper focuses on AUG L-mode relevant parameters. To identify the contributions from different heat flux channels to $\lambda_{q}$ and to gain further understanding for the physical processes determining the scrape-off layer power fall-off length, numerical simulations are employed. The advantage of simulations is that every parameter is known exactly, and thus contributions to $\lambda_{q}$ can be identified and held up against experiments to validate the results. Several papers have investigated how $\lambda_{q}$ scales with different parameters using numerical simulations [11-14], but these studies used either steady-state transport codes or turbulence codes assuming cold ions. In the present paper we investigate the parameter dependence of $\lambda_{q}$ by means of numerical simulations using the HESEL model [15], a 2D drift-fluid model. The model parametrises 3D dynamics with parallel heat fluxes and a sheath connection closure to incorporate the effects along the magnetic field lines as well as drift-wave terms in the region of closed field lines. Although this approach does not capture the full dynamics between the outboard midplane and the divertor, where full 3D codes such as HERMES [16], GBS [17] and TOKAM3X [18] are needed to incorporate these effects, the scaling found in this paper compares well with experimental results found for AUG L-mode [19]. Through turbulence simulations we deduce the parallel losses due to advection and conduction for both electrons and ions, and through parameter scans, with parameters chosen based on statistical significance using an approach similar to that in [3], we calculate numerical scalings for $\lambda_{q}$.

The outline of the paper is as follows; in section 2 we introduce the HESEL model used for the simulations and the methods used for calculating $\lambda_{q}$, in section 3 we present how the scaling parameters are chosen and the derived numerical scaling laws, and finally in section 4 we summarise and conclude the work.

\section{The HESEL model}

The HESEL model [15] is used for simulations throughout the paper. HESEL is a 2D model derived from the Braginskii two-fluid equations [20] solving for the density, $n$, generalised vorticity, $\omega^{*}=\nabla^{2} \varphi+\nabla^{2} p_{i}$, electron pressure, $p_{e}$ and ion pressure, $p_{i}$. 3D dynamics are parametrised following the approach and assumptions given in [21]. The simulations are conducted in a domain at the out-board midplane of a tokamak, as illustrated in Fig. 1. For all equations, Bohm normalisation has been imposed;

$$
\omega_{c i} t \rightarrow t, \quad \frac{\mathbf{x}}{\rho_{s}} \rightarrow \mathbf{x}, \quad \frac{e \varphi}{T_{e 0}} \rightarrow \varphi, \quad \frac{n}{n_{0}} \rightarrow n, \quad \frac{T_{e}}{T_{e 0}} \rightarrow T_{e}, \quad \frac{T_{i}}{T_{e 0}} \rightarrow T_{i},
$$

where $T_{e}$ is the electron temperature, $T_{i}$ is the ion temperature, $n$ is the density, $\varphi$ is the electrostatic potential and $t$ is time. The normalisation parameters are the ion cyclotron frequency, $\omega_{c i}=e B_{0} / m_{i}$, the ion gyroradius at electron temperature, $\rho_{s}=c_{s 0} / \omega_{c i}$, the electron charge, $e$, the reference electron temperature, $T_{e 0}$, the reference density, $n_{0}$,

the magnetic field strength at the outboard mid-plane, $B_{0}$, the ion mass, $m_{i}$, and the constant sound speed at the reference electron temperature, $c_{s 0}=\left(T_{e 0} / m_{i}\right)^{1 / 2}$. 


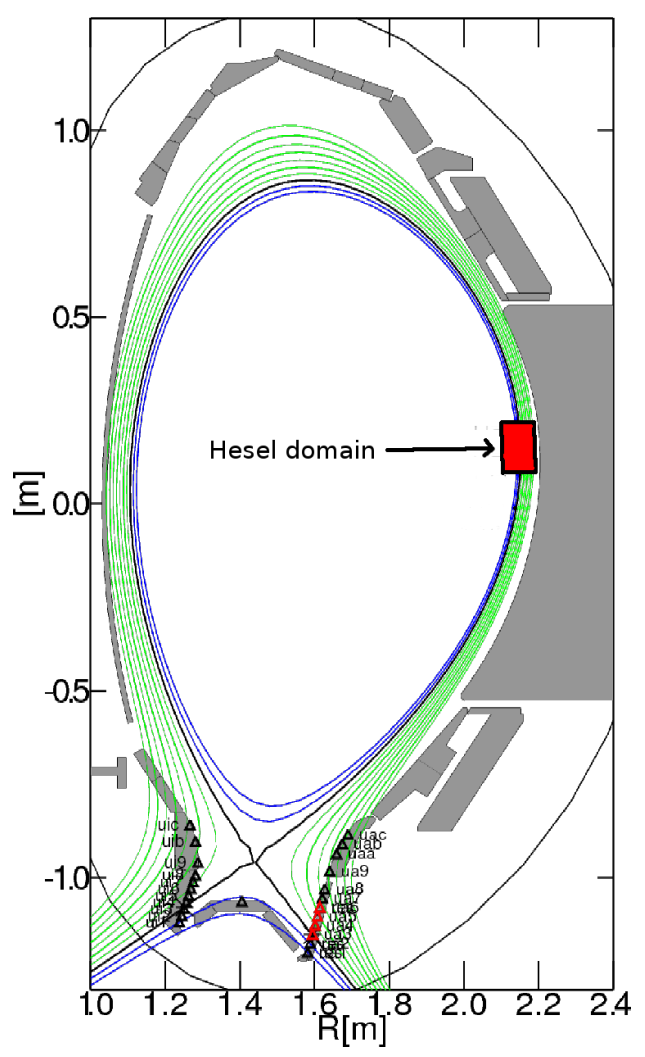

Figure 1: The cross-section of AUG with the HESEL domain indicated to scale by the red box.

The HESEL model reads

$$
\begin{aligned}
& \mathrm{d}_{t} n+n \mathcal{K}(\varphi)-\mathcal{K}\left(p_{e}\right)=\Lambda_{n}, \\
& \mathrm{~d}_{t}^{0} \omega^{*}+\left\{\nabla \varphi, \nabla p_{i}\right\}-\mathcal{K}\left(p_{e}+p_{i}\right)=\Lambda_{\omega^{*}}, \\
& \frac{3}{2} \mathrm{~d}_{t} p_{e}+\frac{5}{2} p_{e} \mathcal{K}(\varphi)-\frac{5}{2} \mathcal{K}\left(\frac{p_{e}^{2}}{n}\right)=\Lambda_{p_{e}}, \\
& \frac{3}{2} \mathrm{~d}_{t} p_{i}+\frac{5}{2} p_{i} \mathcal{K}(\varphi)+\frac{5}{2} \mathcal{K}\left(\frac{p_{i}^{2}}{n}\right)-p_{i} \mathcal{K}\left(p_{e}+p_{i}\right)=\Lambda_{p_{i}},
\end{aligned}
$$

where $\mathrm{d}_{t}=\left(\partial_{t}+B(x)^{-1} \hat{\mathbf{z}} \times \nabla \varphi \cdot \nabla\right)$ is a material derivative, with $B(x)=B_{0}\left(R_{0}+\right.$ $a) /\left(R_{0}+a+x\right)$ being the magnetic field modulus, $R_{0}$ is the device major radius, $a$ is the device minor radius, $x$ is the radial coordinate from the LCFS and $\partial_{t}=\partial / \partial_{t}$. $\mathrm{d}_{t}^{0}=\left(\partial_{t}+B_{0}^{-1} \hat{\mathbf{z}} \times \nabla \varphi \cdot \nabla\right)$ denotes the material derivative with constant magnetic field. $\mathcal{K}=\nabla\left(B(x)^{-1}\right) \cdot \hat{\mathbf{z}} \times \nabla$ is the curvature operator, where $\hat{\mathbf{z}}$ is a unit vector in the direction of the magnetic field. The Poisson bracket is

$$
\{\varphi, f\}=\frac{\partial \varphi}{\partial x} \frac{\partial f}{\partial y}-\frac{\partial f}{\partial x} \frac{\partial \varphi}{\partial y}
$$

The right-hand sides of Eqs. (2)-(5) consist of perpendicular dissipation terms and parametrised parallel dynamics, given by 


$$
\begin{aligned}
& \Lambda_{n}=D_{e} \nabla_{\perp}^{2} n-\frac{n}{\tau_{d}}-\alpha\left(\tilde{T}_{e}+\frac{\tilde{T}_{e}}{\bar{n}} \tilde{n}-\tilde{\varphi}\right)-\frac{n-n_{p}}{\tau_{p}} \\
& \Lambda_{\omega^{*}}=D_{i} \nabla_{\perp}^{2} \omega^{*}-\frac{\omega^{*}}{\tau_{\omega}}+\frac{\rho_{s}}{L_{\|}}\left[1-\exp \left(\varphi_{w}-\frac{\varphi_{s}}{T_{e, s}}\right)\right]-\alpha\left(\tilde{T}_{e}+\frac{\tilde{T}_{e}}{\bar{n}} \tilde{n}-\tilde{\varphi}\right) \\
& \Lambda_{p_{e}}=\frac{5}{2} D_{e} \nabla_{\perp}^{2} p_{e}+\left(\frac{16}{6}-\frac{5}{2} \tau\right) \nabla \cdot\left(n \nabla_{\perp} T_{e}\right)-\frac{9}{2} \frac{p_{e}}{\tau_{d}}-\frac{T_{e}}{\tau_{S H, e}} \\
& \quad-\alpha \tilde{T}_{e}\left(\tilde{T}_{e}+\frac{\tilde{T}_{e}}{\bar{n}} \tilde{n}-\tilde{\varphi}\right)-\Theta-\frac{p_{e}-p_{e, p}}{\tau_{p}} \\
& \Lambda_{p_{i}=} D_{i} \nabla_{\perp}^{2} p_{i}-D_{i} T i \nabla_{\perp}^{2} n-\frac{9}{2} \frac{p_{i}}{\tau_{d}}+\Theta-\frac{p_{i}-p_{i, p}}{\tau_{p}}-\frac{T_{i}}{\tau_{S H, i}}+p_{i} \Lambda_{\omega^{*}},
\end{aligned}
$$

with the neoclassical diffusion coefficients given by

$$
\begin{aligned}
& D_{i}=\left(1+\frac{R_{0}}{a} q^{2}\right) \frac{\rho_{i 0}^{2} \nu_{i i 0}}{\rho_{s}^{2} \omega_{c i}}, \\
& D_{e}=(1+\tau)\left(1+\frac{R_{0}}{a} q^{2}\right) \frac{\rho_{e 0}^{2} \nu_{e i 0}}{\rho_{s}^{2} \omega_{c i}},
\end{aligned}
$$

as derived in [21] where $\rho_{i 0}$ is the ion gyroradius and $\rho_{e 0}$ is the electron gyroradius both at reference temperatures. $\nu_{i i 0}$ is the ion-ion collision frequency at reference temperature, $\nu_{e i 0}$ is the electron-ion collision frequency at reference temperature, $q$ is the safety factor, and $\tau=T_{i 0} / T_{e 0}$, where $T_{i 0}$ is a reference ion temperature. $L_{\|}$is the parallel connection length, $\varphi_{w}=\ln \left(\sqrt{m_{i} /\left(2 \pi m_{e}\right)}\right)$ is the Bohm potential and $\alpha=2 T_{e 0} /\left(\nu_{e i 0} m_{e} L_{\|}^{2} \omega_{c i}\right)$ is the drift-wave coefficient. $\varphi_{s}$ is the potential at the sheath entrance and $T_{e, s}$ is the electron temperature at the sheath entrance. In this paper we have assumed that the plasma is connected to the sheath, which is modelled using $T_{e, s}=T_{e}(x, y, t)$ and $\varphi_{s}=\varphi(x, y, t)$, i.e. the sheath dissipation depends on the full temperature and potential fields.

The term $\Theta=3\left(m_{e} / m_{i}\right) \nu_{e i 0}\left(p_{e}-p_{i}\right)$ is the energy transfer between electrons and ions. The tilde $\tilde{f}$ denotes a fluctuating term of the field $f$ and $\bar{f}$ denotes the poloidal average, defined as

$$
\tilde{f} \equiv \bar{f}-f, \quad \bar{f} \equiv \frac{1}{L_{y}} \int_{0}^{L_{y}} f \mathrm{~d} y,
$$

where $L_{y}$ is the poloidal length of the domain. At the inner boundary, the profiles are forced with the characteristic time $\tau_{p}$ towards prescribed profiles, $n_{p}, p_{e, p}, p_{i, p}$. $\tau_{p}$ is kept shorter than the interchange time, and the profiles are made to resemble typical profiles measured in the edge region of tokamak experiments and thus act as particle and energy sources, while avoiding unphysical steep gradients generated at the inner boundary when using constant particle and energy sources [15].

The parallel dynamics are parametrised in the form of parallel loss terms due to advection and electron and ion heat conduction (Spitzer-Härm conduction) with loss 


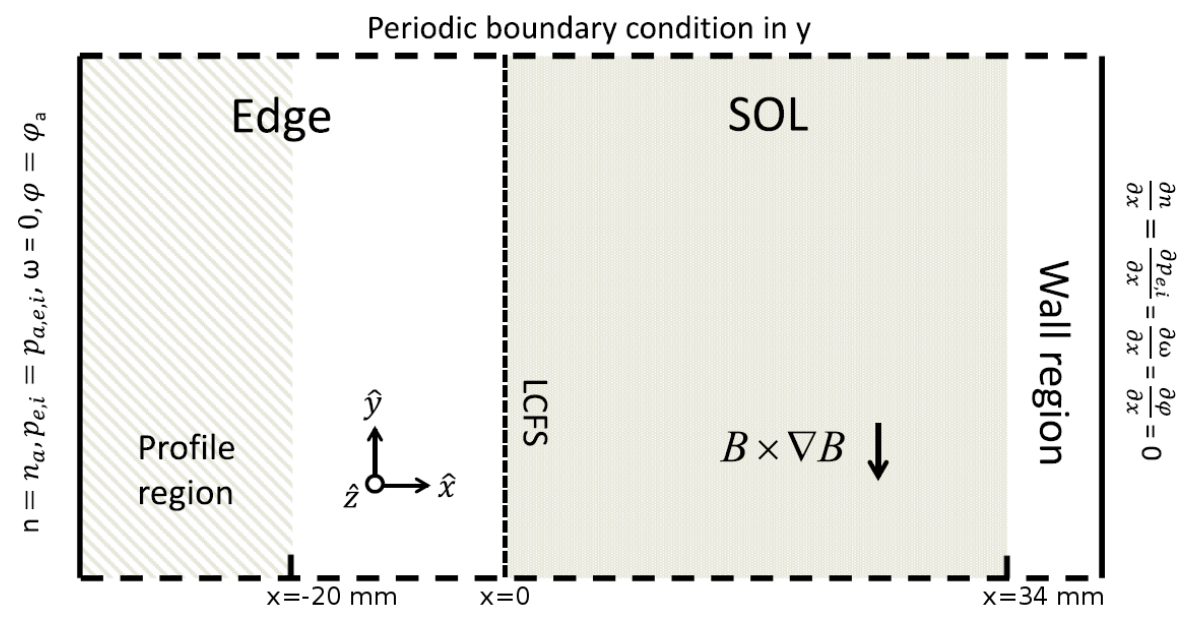

Figure 2: An illustration of the HESEL domain (not to scale) with boundary conditions and geometry illustrated in the plot.

rates given by

$$
\tau_{S H, e}=\frac{L_{B}^{2} \nu_{e e}}{3.2 v_{e}^{2}}, \quad \tau_{S H, i}=\frac{L_{B}^{2} \nu_{i i}}{3.2 v_{i}^{2}}, \quad \tau_{d}=\frac{L_{B}}{2 M c_{s}},
$$

where $L_{B}=q R_{0}$ is the parallel ballooning length. $v_{e(i)}$ is the electron (ion) thermal speed, $\nu_{e e(i i)}$ is the electron-electron (ion-ion) collision frequency, both depending on the local values of $T_{e(i)}$ so that $\tau_{S H, e(i)}^{-1} \propto T_{e(i)}^{5 / 2}$ and $M$ is the parallel Mach number assumed to be $M=0.5$ at the outboard mid-plane (see [22] for a thorough description of this assumption). Finally $c_{s}=\sqrt{\left(T_{e}+T_{i}\right) / m_{i}}$ is the sound speed, which depends on the local electron and ion temperatures. We note that in order for Spitzer-Härm conduction to be valid, the electron collisionality [23]

$$
\nu_{e}^{*} \equiv \frac{L_{\|} \nu_{e i}}{v_{e}} \gg 10
$$

which is fulfilled for all simulations in this paper.

\subsection{Evaluation of $\lambda_{q}$}

Assuming that the transport across the LCFS originates from ballooning-like turbulence in a poloidal extend of $60^{\circ}$ at the out-board mid plane [15], given approximately by $2 \pi a / 6 \approx a$, and assuming that the power is evenly distributed in this region, we can calculate the radial profile of the parallel heat fluxes. This is done using data from from synthetic probes in the HESEL domain illustrated in Fig. 2, where $n_{a}, p_{a, e, i}$ is the value of the fixed profile at the inner boundary for the density and the electron and ion pressures, respectively, and $\varphi_{a}$ (see Fig. 2) is chosen so that $\omega^{*}=0$ at the inner boundary.

The parallel heat flux at the outboard mid-plane is calculated from the parametrised parallel dynamics, and following the parametrisation stated in [21] as well as the contribution from the ion Spitzer-Härm conduction, we get four contributions to the 
parallel heat flux. The approach used in HESEL assumes quasineutrality, which consequently means that only small variation between the parallel velocities is allowed, and as a result, the thermal heat flux is assumed small compared to the other four contributions and has been neglected. The contributions to the parallel heat flux are thus a contribution from the electron advection, $q_{\|, a, e}$, the ion advection, $q_{\|, a, i}$, the electron conduction (also known as Spitzer-Härm conduction), $q_{\|, S H, e}$ and the ion conduction, $q_{\|, S H, i}$, given by

$$
\begin{aligned}
q_{\|, a, e} & =a\left\langle\frac{9}{2} \frac{p_{e}}{\tau_{d}}\right\rangle_{t}, & q_{\|, a, i} & =a\left\langle\frac{9}{2} \frac{p_{i}}{\tau_{d}}\right\rangle_{t} \\
q_{\|, S H, e} & =a\left\langle\frac{p_{e}}{\tau_{S H, e}}\right\rangle_{t}, & q_{\|, S H, i} & =a\left\langle\frac{p_{i}}{\tau_{S H, i}}\right\rangle_{t},
\end{aligned}
$$

where $\langle\cdot\rangle_{t}$ denotes a temporal average. All terms are multiplied by the poloidal extent of the ballooning region, $a$, following the assumption above. We should state that macroscopic heat fluxes have been neglected since the mean fluid velocity at the outboard mid-plane is negligible. However, when approaching the divertor target, part of the thermal energy in expanding filaments will be converted to kinetic energy of the ions streaming toward the target plates at the filament fronts and this contribution to the heat flux would have to be evaluated. However, at the outboard mid-plane the parallel heat flux is described by the four contributions stated above. It is important to note that the full temperature and pressure fields are used for computing the parallel fluxes in Eqs. (16) and (17). Since a significant fraction of the transport across the LCFS is intermittent [24], the parallel heat fluxes can not be calculated solely based on temporally averaged profiles, but need to be evaluated using the full temporal signals to capture the contributions from the intermittent bursts. To illustrate the importance of using the full fields, we have plotted the electron temperature at the LCFS in the poloidal centre of the domain as a function of time in Fig. 3a. Here it is seen that the electron temperature is subject to large fluctuations varying by more than a factor of five. This inevitably influences the value of the parallel heat flux, as is illustrated in Fig. $3 \mathrm{~b}$, where we have plotted the electron contributions to the parallel heat flux evaluated using Eqs. (16) and (17) using the full fields (blue) and the temporally averaged fields (magenta). The difference is most pronounced in the Spitzer-Härm conduction, where the averaged profile leads to an estimation of the heat flux, which is a factor of two smaller at the LCFS than the profile found using the full fields.

The total parallel heat flux is the sum of all four contributions in Eqs. (16) and (17), so

$$
q_{\|}=q_{\|, a, e}+q_{\|, a, i}+q_{\|, S H, e}+q_{\|, S H, i}
$$

The scrape-off layer power fall-off length is then calculated as

$$
\lambda_{q}=\frac{\int_{0}^{\infty} x q_{\|}(x) \mathrm{d} x}{\int_{0}^{\infty} q_{\|}(x) \mathrm{d} x},
$$

where 0 is the location of the separatrix. 


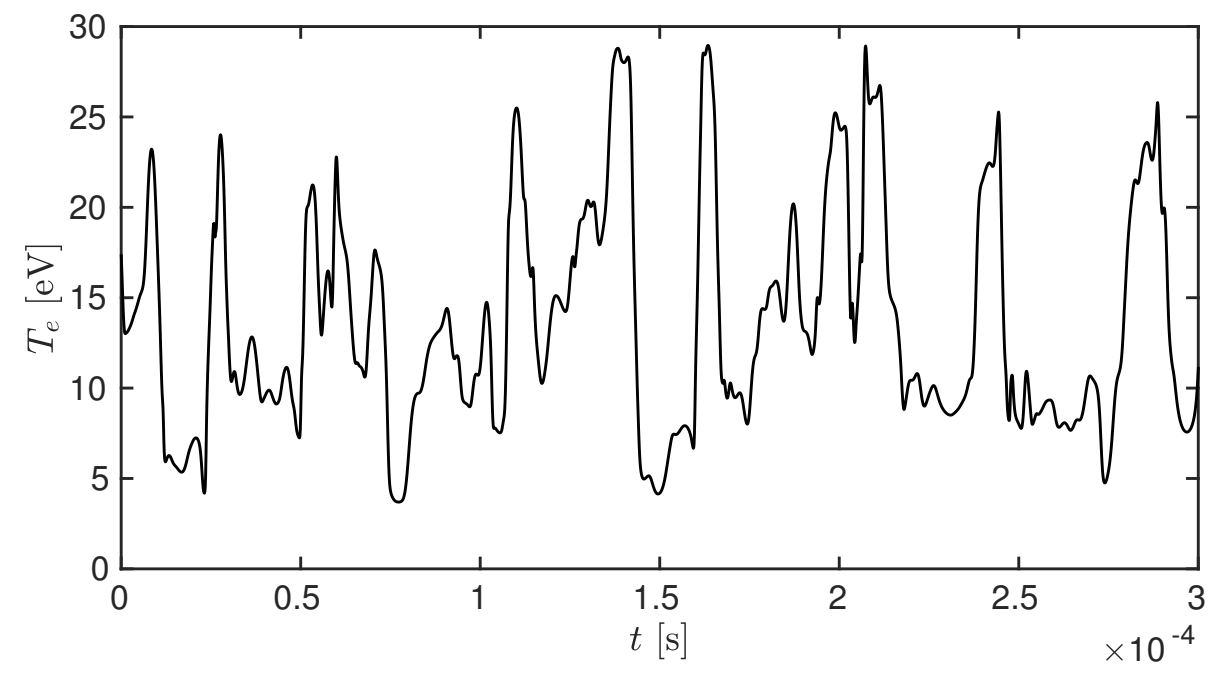

(a)

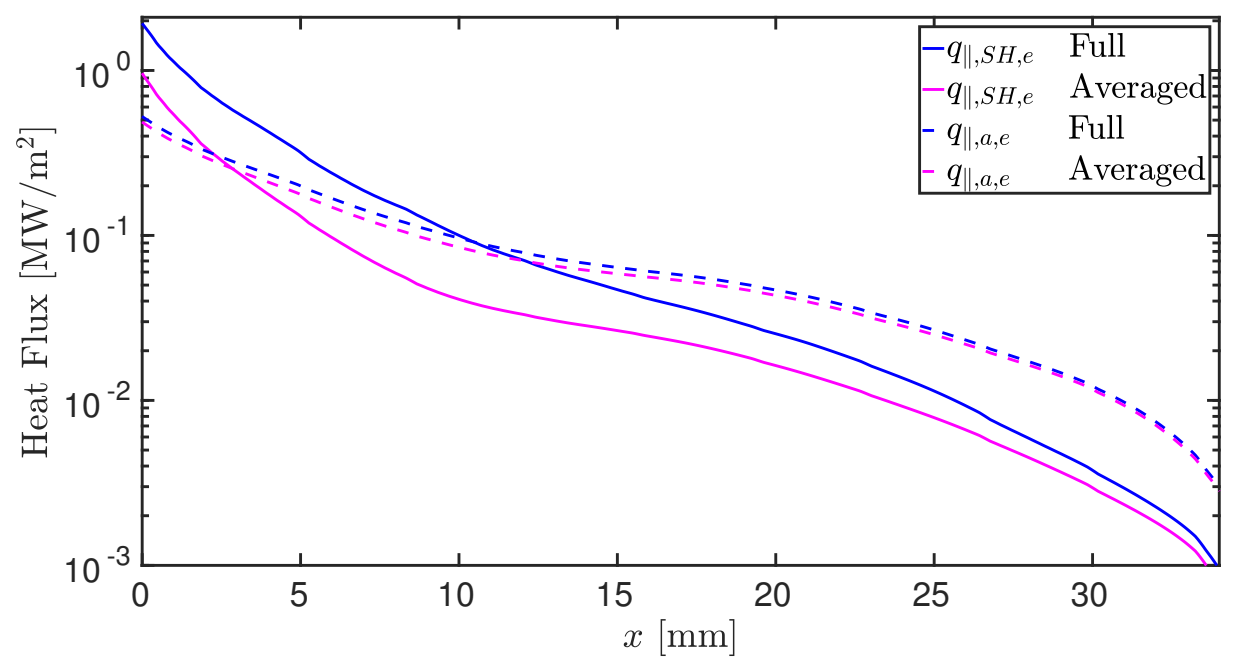

(b)

Figure 3: (a) Fluctuation level at the LCFS in the poloidal centre of the domain for a simulation with AUG relevant parameters. Only part of the temporal domain is shown. The full simulation is an order of magnitude longer. (b) The electron conduction (solid) and electron advection (dashed) contributions to the parallel heat flux for a typical simulation with AUG relevant parameters. The blue lines indicate the contributions calculated using the full fields and the magenta lines indicate the contributions calculated using averaged profiles. 


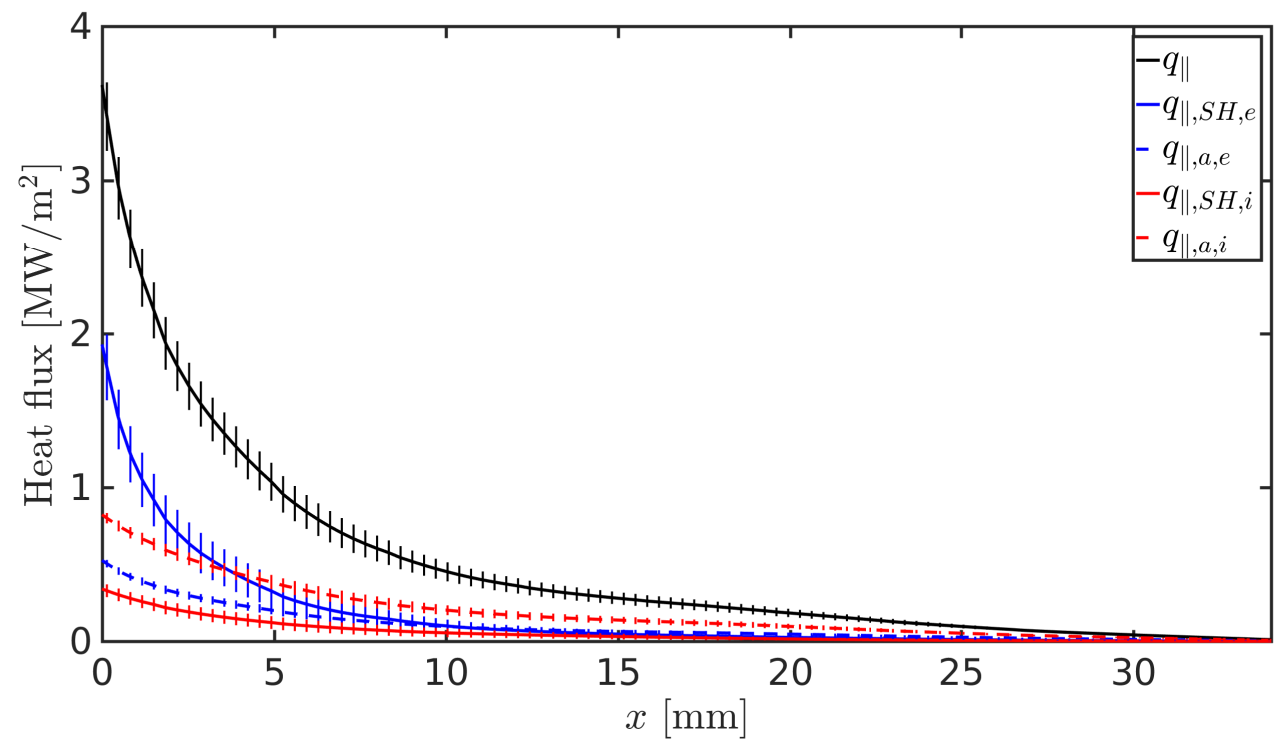

Figure 4: A typical parallel heat flux profile for AUG relevant parameters. The vertical lines indicate the $95 \%$ confidence limits on the averaged values.

A typical heat flux profile for parameters relevant for ASDEX Upgrade is seen in Fig. 4. The four contributions to the total heat flux are illustrated in the plot, and as seen from the figure, the electron conduction dominates the parallel transport close to the LCFS, while the ion advection and electron advection become the dominant terms in the far SOL, where the ion conduction remains insignificant throughout the SOL. This is made more evident when looking at the ratio of the individual contributions with respect to the full heat flux profile as a function of the radial position, which is illustrated in Fig. 5. Here we see that the contribution from ion advection becomes dominant a few millimetres into the SOL. Note that the illustrated domain does not show the full simulation domain, but only depicts the SOL.

\section{Scrape-off layer power fall off length}

The simulations, from which the heat flux profiles are calculated are repeated scanning several AUG relevant L-mode parameters. This is done in order to derive a numerical scaling law for $\lambda_{q}$ in L-mode. $\lambda_{q}$ is calculated using Eq. (19) for every scanned parameter, and a fit of $\lambda_{q}$ is made as a function of different combinations of parameters. The scans are performed by varying the parameters one at a time while all other parameters are kept fixed at typical AUG L-mode values, $R_{0}=1.65 \mathrm{~m}, a=0.5 \mathrm{~m}, q=4.5$ and $B_{0}=1.9 \mathrm{~T}$. Since the total power across the LCFS, $P$, and the LCFS values of $n, T_{e}$ and $T_{i}$ are not input parameters, but depend on the forced profiles and the scanned input parameters, they vary slightly when the other parameters are scanned and they themselves are scanned by varying their respective forced profiles. The range of the scans are listed in table 1. 


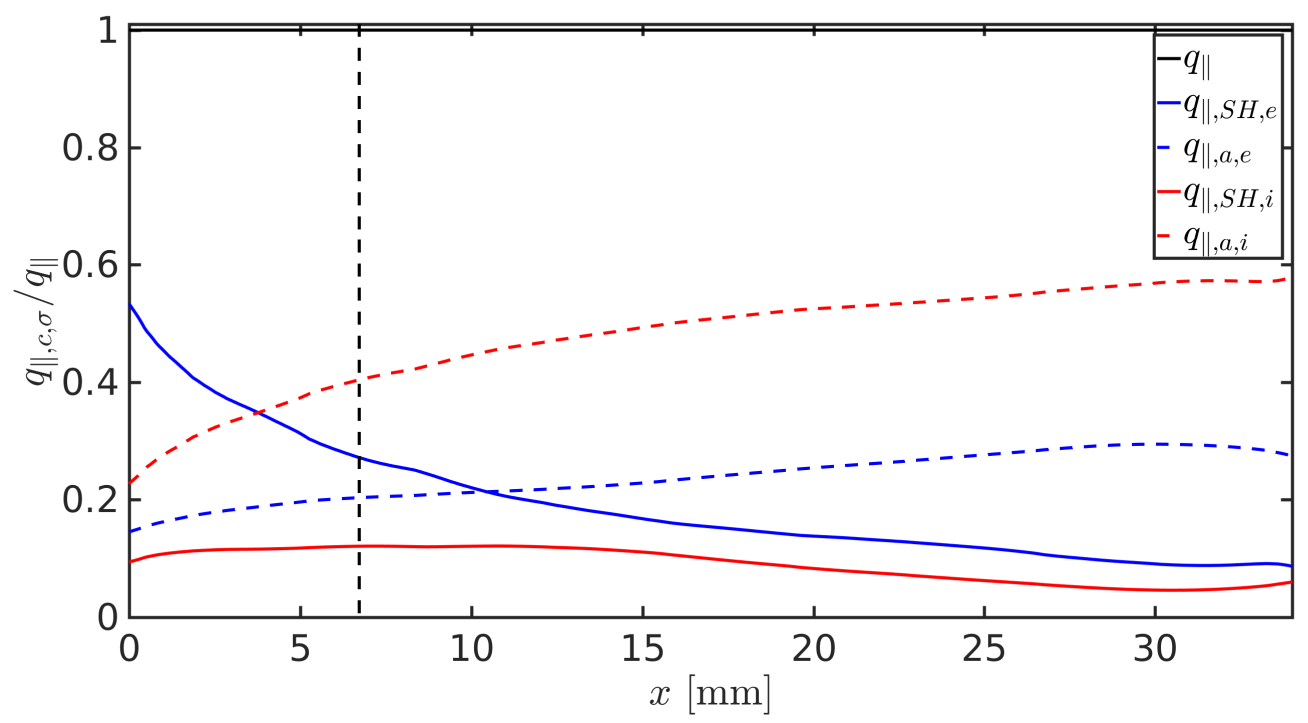

Figure 5: The fraction of the total heat flux for each of the four contributions. The dashed black line indicates the extent of the power fall-off length, $\lambda_{q}$, as calculated using Eq. (19).

Table 1: The scaled parameters with corresponding ranges.

\begin{tabular}{llr}
\hline Parameter & units & range \\
\hline$R_{0}$ & {$[\mathrm{~m}]$} & $1-5$ \\
$a$ & {$[\mathrm{~m}]$} & $0.3-1.2$ \\
$q$ & & $3.8-9.0$ \\
$B_{0}$ & {$[\mathrm{~T}]$} & $1.5-2.3$ \\
$P$ & {$[\mathrm{MW}]$} & $0.04-1.27$ \\
$n_{L C F S}$ & {$\left[10^{19} \mathrm{~m}^{3}\right]$} & $1.1-2.8$ \\
$T_{e, L C F S}$ & {$[\mathrm{eV}]$} & $11-22$ \\
$T_{i, L C F S}$ & {$[\mathrm{eV}]$} & $14-43$ \\
\hline
\end{tabular}

Here $P=2 \pi\left(R_{0}+a\right) \int_{0}^{\infty} q_{\|}(x) \mathrm{d} x$ is the total power across the LCFS, where the $2 \pi\left(R_{0}+a\right)$ accounts for the circumference of the tokamak, $n_{L C F S}$ is the density at the LCFS and $T_{e(i), L C F S}$ is the electron(ion) temperature at the LCFS. We are aware that the electron and ion temperatures are low compared to usual AUG parameters, however, it should be noted that the LCFS is at the bottom of a steep gradient and moving the position $0.5 \mathrm{~cm}$ further in (which is less than the precision with which the position of the LCFS is determined in experiments) increases the temperatures by more than $50 \%$.

\subsection{Comparison with experimental scaling}

First we investigate the experimental L-mode scaling from AUG derived in [19]. In this paper, a scan of $P$ and $q$ is performed, while the fit of $B_{0}$ is taken from a study of an 


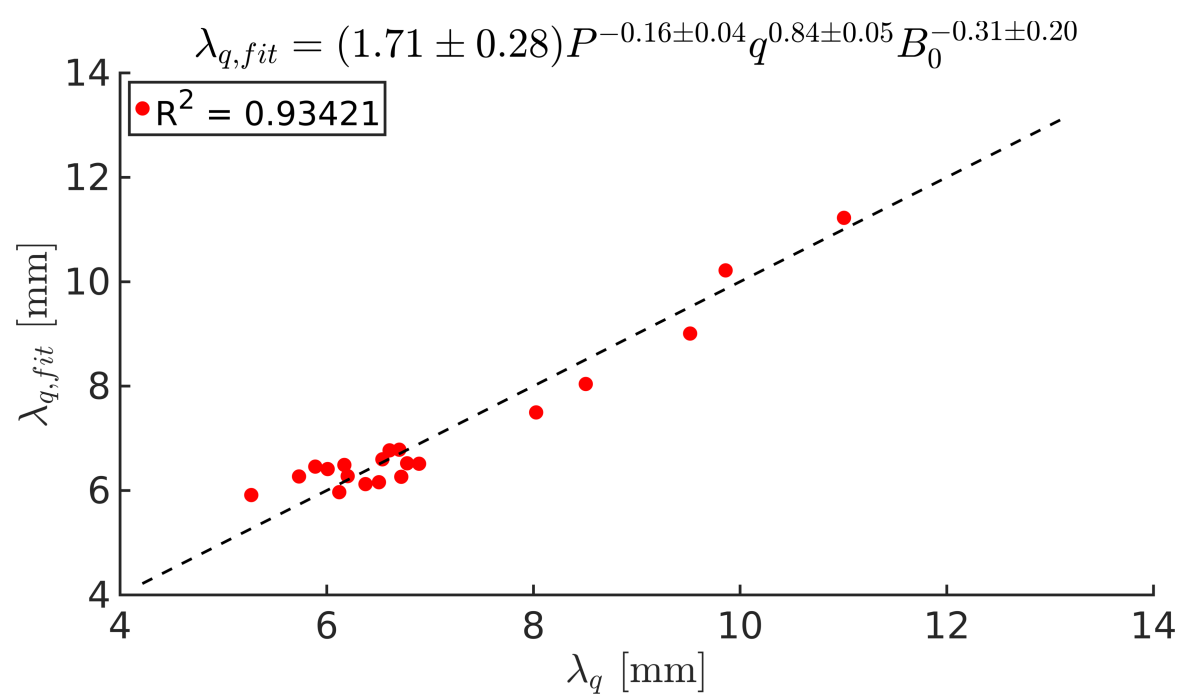

Figure 6: The nonlinear fit of $\lambda_{q}$ with the parameters $P, q$ and $B_{0}$ plotted with respect to the numerically found $\lambda_{q}$, given by Eq. (19). Note that each dot corresponds to a separate simulation.

H-mode scaling at AUG in [5] and the parameter was thus not scanned in the paper. The scaling arrived at is given by

$$
\lambda_{q, \text { Sieg }}=(1.45 \pm 0.38) P^{-0.14 \pm 0.10} q^{1.07 \pm 0.07} B_{0}^{-0.78},
$$

where $B_{0}$ is highlighted in red since it is not a scanned parameter.

In order to compare our numerical results with the experiments, we include scans with the same parameters as in [19]. This means that $R_{0}=1.65 \mathrm{~m}$ and $a=0.5 \mathrm{~m}$ are kept constant, while the variation in $n_{L C F S}$ is kept small using a range of $1.2 \times 10^{19} \mathrm{~m}^{3}$ to $1.4 \times 10^{19} \mathrm{~m}^{3}$. A fit is then made using

$$
\lambda_{q, f i t}=A P^{b} q^{c} B_{0}^{d}
$$

where a nonlinear fitting routine using an iterative least squares estimation (nlinfit in MATLAB) is used to determine the parameters $A, b, c$ and $d . \quad \lambda_{q, f i t}$ is plotted as a function of the numerically found value for $\lambda_{q}$ in Fig. 6 , where the best fit is found to be

$$
\lambda_{q, f i t}=(1.71 \pm 0.28) P^{-0.16 \pm 0.04} q^{0.84 \pm 0.05} B_{0}^{-0.31 \pm 0.20} .
$$

We observe a fit with a coefficient of determination of $R^{2}=0.93$. The fit is weakly dependent on the power across the last closed flux surface, $P$, it scales almost linearly with the safety factor, $q$, and it is weakly dependent on the toroidal magnetic field, $B_{0}$. Comparing this scaling to Eq. (20) we observe a match within the error bars on $P$ and we observe the same trend with an almost linear dependence with $q$. The scaling with toroidal magnetic field, $B_{0}$, differs between the two scalings, however, it should be noted that $B_{0}$ was not a scaled parameter in [19], so the two scalings cannot be directly compared with respect to this parameter. 


\subsection{Choice of scaling parameters}

The scaling in the previous section was derived in order to compare the results with the experimental scaling from AUG. This meant that the scanned parameters were limited to $P, q$ and $B_{0}$. Now, in order to make a more systematic investigation of the parameter dependence of $\lambda_{q}$, we fit it to all parameters listed in Tab. 1. However, some of the parameters have strong mutual correlation, so, following the approach in [3], we calculate the correlation between the different parameters. Since the correlation coefficient describes a linear correlation between two quantities, we determine the coefficient by making a linear fit between the logarithms of the two parameters in question. The mutual correlation between each parameter is illustrated in Tab. 2, and any parameters with a correlation of more than $50 \%$ are highlighted in bold face and are not included in the same scalings. We observe that the total power across

Table 2: Mutual correlation between the logarithms of the different fitting parameters.

\begin{tabular}{|l|l|l|l|l|l|l|l|l|}
\hline Corr. (\%) & $a$ & $n_{\text {LCFS }}$ & $q$ & $T_{e, L C F S}$ & $T_{i, L C F S}$ & $B_{0}$ & $P$ & $a / R_{0}$ \\
\hline$R_{0}$ & $\mathbf{6 3}$ & 24 & 0 & 49 & 19 & 1 & $\mathbf{7 1}$ & 0 \\
$a$ & & 24 & 0 & 35 & 15 & 1 & $\mathbf{7 9}$ & 9 \\
$n_{L C F S}$ & & & 7 & 23 & 12 & 3 & $\mathbf{5 3}$ & 0 \\
$q$ & & & 37 & 32 & 1 & 6 & 13 \\
$T_{e, L C F S}$ & & & & & $\mathbf{8 9}$ & 5 & $\mathbf{7 6}$ & 0 \\
$T_{i, L C F S}$ & & & & & & 6 & $\mathbf{5 0}$ & 0 \\
$B_{0}$ & & & & & & & 5 & 0 \\
$P$ & & & & & & & & 0 \\
\hline
\end{tabular}

the LCFS depends on a wide variety of scaling parameters, so in order to get a better understanding of what determines $\lambda_{q}$, it is fruitful to make a fit, where $P$ is neglected and the other mutually independent parameters are included instead. Revisiting the scaling in Eq. (22) where $P$ is replaced with the parameters $n_{L C F S}$ and $T_{e, L C F S}$, we again apply the nonlinear fitting routine to get $\lambda_{q, f i t}$. All parameter exponents in the fit are subject to uncertainties and are accompanied with $95 \%$ confidence intervals as calculated by the fitting routine. If the interval encompasses 0 , then the parameter has no statistical significance and can be omitted from the scaling. When a parameter is omitted the fit is redone without this parameter and the process is repeated until only parameters with statistical significance remain. The outcome of this iterative routine is shown in Tab. 3. We observe a very good fit with a coefficient of determination of $R^{2}=0.96$ when including all parameters, which leads to a scaling given by

$$
\lambda_{q, f i t}=(8.06 \pm 2.14) n_{L C F S}^{0.54 \pm 0.32} q^{0.85 \pm 0.07} B_{0}^{-0.20 \pm 0.15} T_{e, L C F S}^{-0.58 \pm 0.11},
$$

as illustrated in Fig. 7. However, we can, without significantly deteriorating the coefficient of determination, remove the parameters with the largest uncertainties, which is what we have done in the last two iterations in Tab. 3. This leads to a simple scaling, 
Table 3: Scaling where $P, q$ and $B_{0}$ are scanned.

\begin{tabular}{|l|l|l|l|l|l|}
\hline$A$ & $n_{L C F S}$ & $q$ & $B_{0}$ & $T_{e, L C F S}$ & $R^{2}$ \\
\hline $8.06 \pm 2.14$ & $0.54 \pm 0.32$ & $0.85 \pm 0.07$ & $-0.20 \pm 0.15$ & $-0.58 \pm 0.11$ & 0.96 \\
$6.16 \pm 1.38$ & - & $0.93 \pm 0.05$ & $-0.28 \pm 0.15$ & $-0.45 \pm 0.08$ & 0.96 \\
$5.02 \pm 1.04$ & - & $0.92 \pm 0.05$ & - & $-0.44 \pm 0.09$ & 0.95 \\
\hline
\end{tabular}

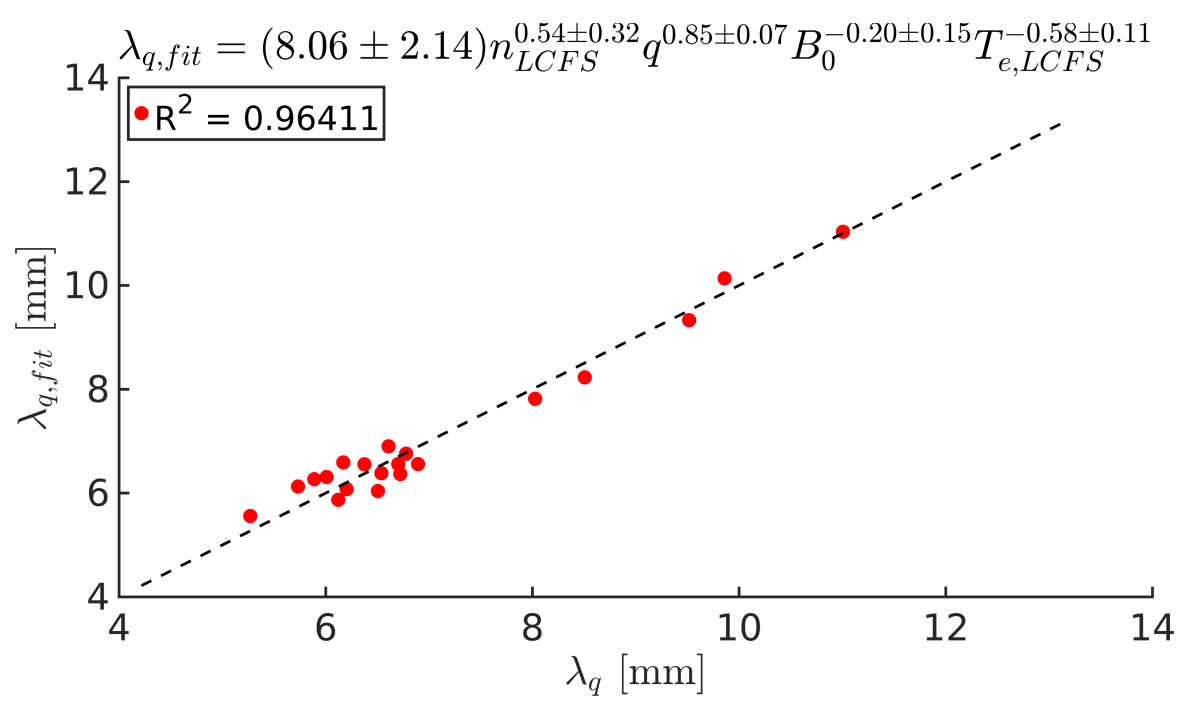

Figure 7: The nonlinear fit $\lambda_{q}$ with respect to the parameters $n_{L C F S}, q, B_{0}$ and $T_{e, L C F S}$ for simulations where $P, q$ and $B_{0}$ are scanned.

where $\lambda_{q}$ is solely determined by the electron temperature at the LCFS and the safety factor;

$$
\lambda_{q, f i t}=(5.02 \pm 1.04) q^{0.92 \pm 0.05} T_{e, L C F S}^{-0.44 \pm 0.09}
$$

with a coefficient of determination of $R^{2}=0.95$, which is plotted in Fig. 8. Comparing this fit, to Fig. 7, we do not observe any major improvements to the fit quality with more parameters. This means that the extra parameters in the fit in Eq. (23) compared to Eq. (24) merely add complexity, but do not significantly improve how well $\lambda_{q}$ is described by the parameters.

\subsection{Scaling with $n_{L C F S}$}

The $\lambda_{q}$ scalings found in Eqs. (22) and (24) were derived with a scan over $P, q$ and $B_{0}$ to be able to compare with experimentally found values. However, we observed large uncertainties on the exponents of several parameters in the scalings, so in order to reduce the range of the confidence interval for the parameter exponents, a dedicated scan of $n_{L C F S}$ with the range given in Tab. 1 was conducted and included in the $\lambda_{q}$ fits. The nonlinear fitting routine described previously is applied with the extra parameter scan and the result is seen in Tab. 4. We get a match within the errorbars on all parameters compared to the fit in seen in Eq. (23). However, we still get large uncertainties on $B_{0}$ 


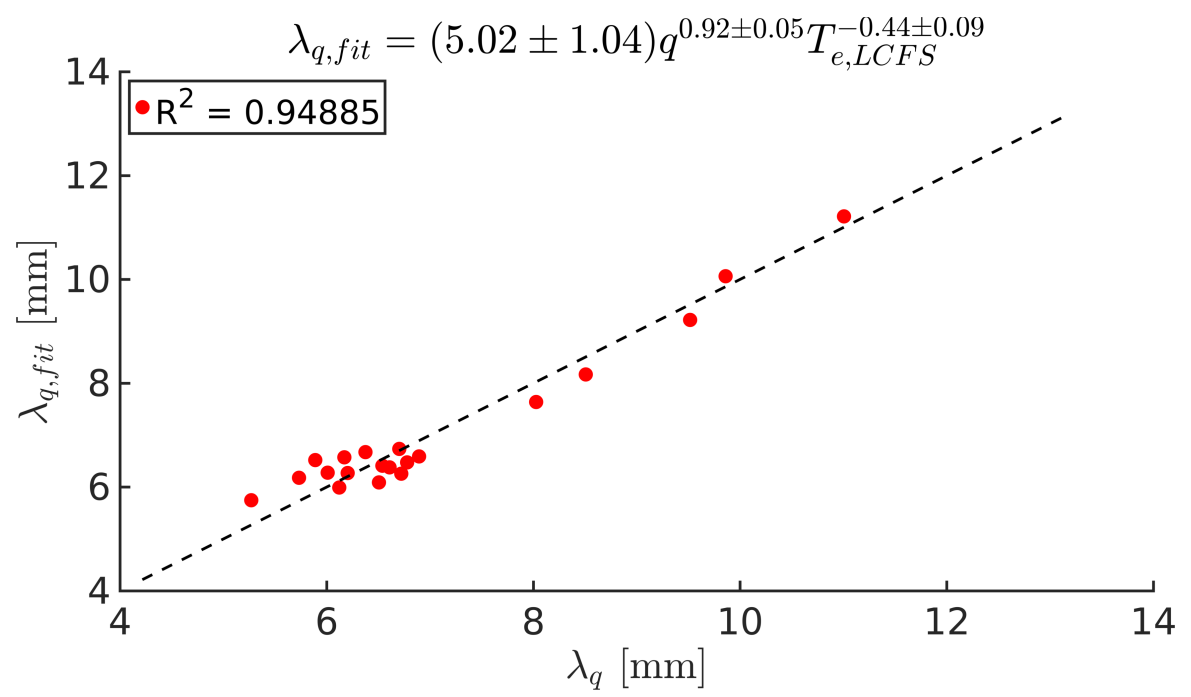

Figure 8: The nonlinear fit $\lambda_{q}$ with respect to the parameters $T_{e, L C F S}$ and $q$ for simulations where $P, q$ and $B_{0}$ are scanned.

Table 4: Scaling where $P, q, B_{0}$ and $n_{L C F S}$ are scanned.

\begin{tabular}{|l|l|l|l|l|l|}
\hline$A$ & $n_{L C F S}$ & $q$ & $B_{0}$ & $T_{e, L C F S}$ & $R^{2}$ \\
\hline $5.57 \pm 1.28$ & $0.23 \pm 0.05$ & $0.86 \pm 0.05$ & $-0.23 \pm 0.18$ & $-0.41 \pm 0.08$ & 0.93 \\
$4.74 \pm 0.92$ & $0.24 \pm 0.05$ & $0.86 \pm 0.05$ & - & $-0.40 \pm 0.08$ & 0.93 \\
\hline
\end{tabular}

and when removing this parameter from the scaling, as is done in the last step in Tab. 4 , we do not observe a significant reduction in the fit quality. This leads to a fit given by

$$
\lambda_{q, f i t}=(4.74 \pm 0.92) n_{L C F S}^{0.24 \pm 0.05} q^{0.86 \pm 0.05} T_{e, L C F S}^{-0.40 \pm 0.06}
$$

with a coefficient of determination of $R^{2}=0.93$. The fit is plotted in Fig. 9 and we observe that the points lie very close to the straight line indicating that $\lambda_{q}$ is described well by the fourth root of $n_{L C F S}$, which compares well with [6], the inverse square root of $T_{e, L C F S}$ and linearly with $q$.

\subsection{Scaling with $R_{0}$}

Finally, we investigate the dependence of $\lambda_{q}$ on the major radius, where all parameter scans from Tab. 1 are included. $R_{0}$ enters the calculations in a wide range of ways. First it enters in the curvature operator $\propto 1 / R_{0}$, so a larger major radius decreases the amount of turbulence. The connection lengths and ballooning lengths are $\propto R_{0}$ and the neoclassical corrections in the diffusivities are $\propto R_{0} q^{2}$. This means that the perpendicular diffusivities increase with $R_{0}$, whereas the sheath loss and the other parallel losses decrease. It is therefore difficult to predict exactly how $R_{0}$ will affect $\lambda_{q}$.

The parameter scan of $R_{0}$ is conducted both with a fixed aspect ratio, $a / R_{0}$ of 


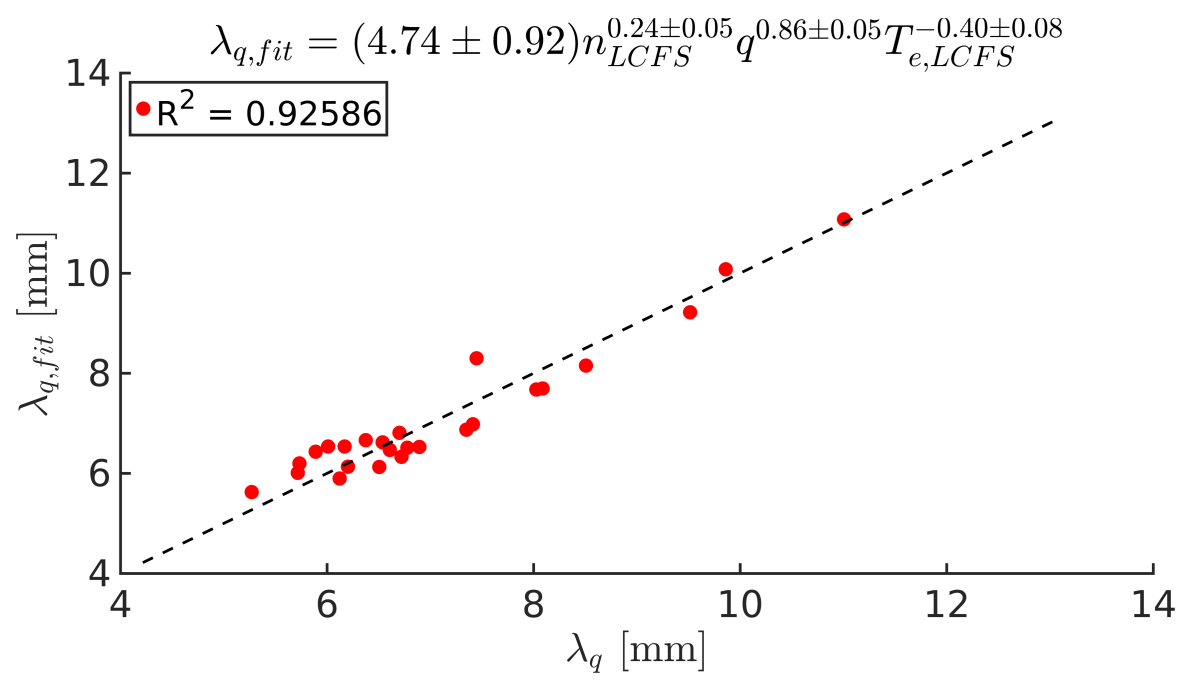

Figure 9: The nonlinear fit of $\lambda_{q}$ with respect to the parameters $n_{L C F S}, q$ and $T_{e, L C F S}$ for simulations where $P, q, B_{0}$ and $n_{L C F S}$ are scanned.

0.3 , varying both $a$ and $R_{0}$ to keep the ratio fixed, and with a fixed minor radius of $a=0.5 \mathrm{~m}$, so the aspect ratio varies between 0.1 and 0.3 . The scaling is seen in Tab. 5 .

Table 5: Scaling where $P, q, B_{0}, n_{L C F S}$ and $R_{0}$ are scanned.

\begin{tabular}{|l|l|l|l|l|l|l|}
\hline$A$ & $n_{L C F S}$ & $q$ & $B_{0}$ & $T_{e, L C F S}$ & $R_{0}$ & $R^{2}$ \\
\hline $3.70 \pm 1.04$ & $0.27 \pm 0.06$ & $0.84 \pm 0.07$ & $-0.21 \pm 0.24$ & $-0.37 \pm 0.10$ & $0.64 \pm 0.04$ & 0.93 \\
$3.19 \pm 0.72$ & $0.27 \pm 0.06$ & $0.84 \pm 0.07$ & - & $-0.36 \pm 0.10$ & $0.63 \pm 0.04$ & 0.93 \\
\hline
\end{tabular}

The best fit is found to be

$$
\lambda_{q, f i t}=(3.19 \pm 0.72) n_{L C F S}^{0.27 \pm 0.06} q^{0.84 \pm 0.07} T_{e, L C F S}^{-0.36 \pm 0.10} R_{0}^{0.63 \pm 0.04},
$$

with $R^{2}=0.93$. As illustrated in Fig. 10, the fit is very close to the straight line with the exception of two outliers for very small major radii. This scaling with the major radius is in sharp contrast to what was found in the multimachine scaling in [5], where $\lambda_{q}$ was found to be independent of the major radius, but agrees well with Halpern [8], Militello [13] and Myra [25]. The discrepancy with the experimental multi machine scaling may be attributed to the decrease in parallel losses with increasing major radius, which broadens $\lambda_{q}$. However, these parallel loss terms are 2D parametrisations of a 3D field, and therefore do not capture the dynamics between the outboard midplane and the divertor region, where the parallel heat flux is measured in [5]. In order to capture these dynamics and describe the heat flux at the divertor, a full 3D model will be necessary. The effects of such 3D models on filamentary transport are discussed in [26] and [27] and the inclusion of 3D effects on the SOL width is discussed in [28]. 


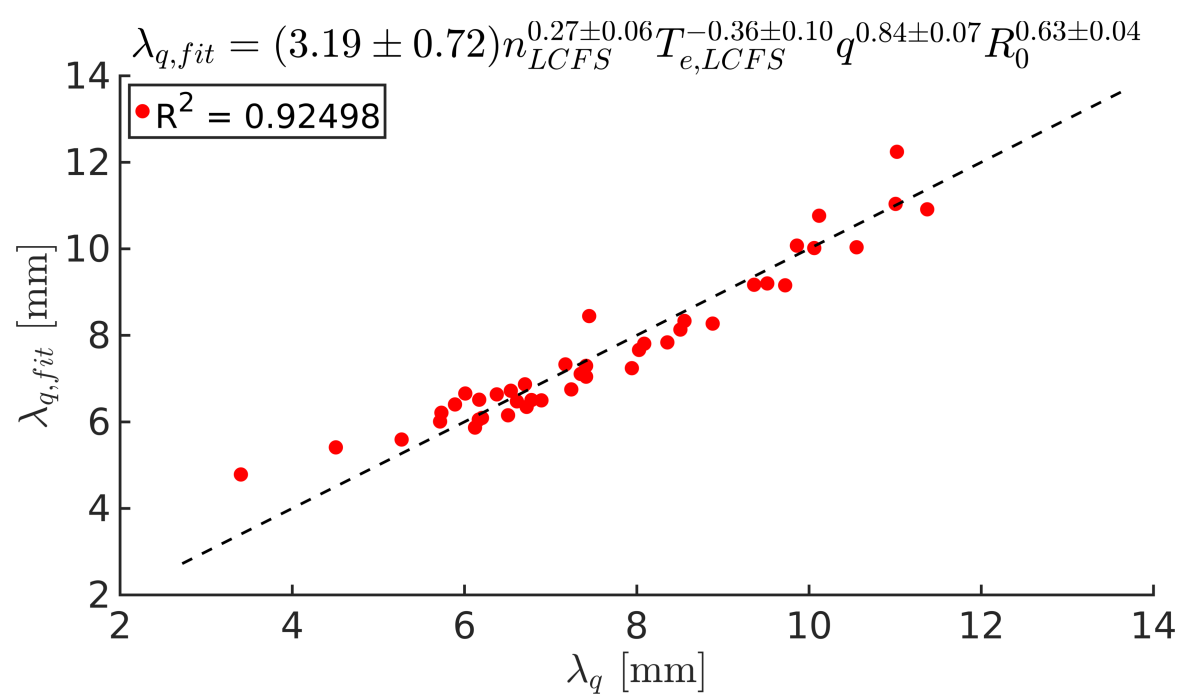

Figure 10: The nonlinear fit of $\lambda_{q}$ with respect to the parameters $n_{L C F S}, q, T_{e, L C F S}$ and $R_{0}$ for simulations where $n, P, q, B_{0}$.

\section{Conclusion}

In this paper, we have investigated the SOL power fall-off length, $\lambda_{q}$, by means of numerical simulations using the HESEL model. Since plasma transport in a tokamak is strongly intermittent, it is crucial to account for this intermittency when determining $\lambda_{q}$ and averaged profiles can thus not be used. We have calculated the SOL power fall-off length by determining the parallel heat fluxes using full fields. Assuming that both advection and conduction for ions and electrons constitute the parallel heat flux, we get four individual contributions. We observe for typical AUG L-mode parameters, that electron conduction dominates the parallel heat flux close to the separatrix, but further into the SOL electron and ion advection take over, with the ion advection being dominant in the far SOL, and broaden $\lambda_{q}$, which is determined by the weighted average position of the parallel heat flux.

In order to compare with experimentally found scaling laws for $\lambda_{q}$, we initially made a parameter scan using parameters comparable to those used in AUG L-mode discharges. From this, we found a scaling law given by

$$
\lambda_{q, f i t}=(1.71 \pm 0.28) P^{-0.16 \pm 0.04} q^{0.84 \pm 0.05} B_{0}^{-0.31 \pm 0.20},
$$

which is close to the L-mode scaling in [19] with a weak dependence on the total power across the LCFS, $P$, and an almost linear dependence on the safety factor, $q$. The dependence on the toroidal magnetic field differs between the experimental and numerical scalings, but since it was not a scaled parameter in [19] we cannot compare the two.

The experimental scaling laws were derived on the basis of engineering parameters, which influence the properties of the plasma, but do not tell us much about the physical processes behind the SOL power fall-off length. To get a better understanding of what 
dictates $\lambda_{q}$, we examined the correlation between parameters which influence $\lambda_{q}$. It was found that $P$ depends on a number of different parameters, so to understand what determines $\lambda_{q}$, the scaling was redone with $P$ replaced by the density at the LCFS, $n_{L C F S}$, and the electron temperature at the LCFS $T_{e, L C F S}$. Here it was found that $\lambda_{q}$ can be described by the two parameters $q$ and $T_{e, L C F S}$ and that $\lambda_{q}$ scales almost linearly with $q$ and as one over the square root of $T_{e, L C F S}$.

Moving on to include a wider range of scanned parameters, including a dedicated scan of $n_{L C F S}$ and the major radius, $R_{0}$, revealed a dependence of $\lambda_{q}$ on $n_{L C F S}$ and $R_{0}$ in addition to the dependence on $q$ and $T_{e, L C F S}$. The best fit for $\lambda_{q}$ was found to be

$$
\lambda_{q, f i t}=(3.19 \pm 0.72) n_{L C F S}^{0.27 \pm 0.06} q^{0.84 \pm 0.07} T_{e, L C F S}^{-0.36 \pm 0.10} R_{0}^{0.63 \pm 0.04} .
$$

The dependence on $R_{0}$ conflicts with what was found in [5], where $\lambda_{q}$ was found to be independent of $R_{0}$. Since the major radius enters the HESEL model both in the parallel loss-terms, the diffusivities and in the curvature operator, it is not straightforward to determine which term this inconsistency arises from and this analysis is therefore left for future studies.

Despite this we do observe a good fit with the experimental L-mode scaling for AUG, and we believe that the $\lambda_{q}$ scalings we have found in this paper can act as a guideline for future experiments. In all scaling laws derived in this paper, it is important to note that the theory used is only valid for strongly collisional plasmas. The extrapolation of $\lambda_{q}$ to ITER, however, needs to be done with care, since ITER is not expected to be dominated by Spitzer-Härm conduction due to it operating in a low collisional regime.

\section{Acknowledgements}

This work has been carried out within the framework of the EUROfusion Consortium and has received funding from the Euratom research and training programme 2014-2018 under grant agreement No. 633053. The views and opinions expressed herein do not necessarily reflect those of the European Commission.

\section{References}

[1] Eich T, Sieglin B, Scarabosio A, Fundamenski W, Goldston RJ, Herrmann A, et al. Inter-ELM power decay length for JET and ASDEX Upgrade: measurement and comparison with heuristic drift-based model. Phys. Rev. Lett. 2011;107(21):215001.

[2] Kocan M, Pitts R A, Arnoux G, Balboa I, de Vries P C, et al. Impact of a narrow limiter SOL heat flux channel on the ITER first wall panel shaping Nucl. Fusion 2015;55:033019.

[3] Horacek J, Pitts R A, Adamek J, Arnoux G, Bak J-G, et al. Multi-machine scaling of the main SOL parallel heat flux width in tokamak limiter plasmas Plasma Phys. Contr. Fusion. 2016;58:074005.

[4] Silva C, Arnoux G, Devaux S, Frigione D, Groth M, et al. Characterization of scrape-off layer transport in JET limiter plasmas Nucl. Fusion. 2014;54:083022.

[5] Eich T, Leonard AW, Pitts RA, Fundamenski W, Goldston RJ, Gray TK, Herrmann A, Kirk A, Kallenbach A, Kardaun O, et al. Scaling of the tokamak near the scrape-off layer H-mode power width and implications for ITER. Nucl. Fusion. 2013;53(9):093031. 
[6] Sieglin B, Eich T, Faitsch M, Herrmann A, Nille D, Scarabosio A, et al. Density dependence of SOL power width in ASDEX upgrade L-Mode Nucl. Mater. Energy. 2017;12:216-220.

[7] Faitsch M, Sieglin B, Eich T, Sun HJ, Herrmann A, et al. Change of the scrape-off layer power width with the toroidal B-field direction in ASDEX upgrade Plasma Phys. Contr. Fusion. 2015;57:075005.

[8] Halpern FD, Horacek J, Pitts RA, Ricci P. A theoretical interpretation of the main scrape-off layer heat-flux width scaling for tokamak inner-wall limited plasmas Plasma Phys. Contr. Fusion. 2016;58(8):084003.

[9] Sun HJ, Wolfrum E, Eich T, et al. Study of near scrape-off layer (SOL) temperature and density gradient lengths with Thomson scattering Plasma Phys. Contr. Fusion. 2015;57:125001.

[10] Eich T, Goldston RJ, Kallenbach A, Sieglin B, et al. Correlation of the tokamak H-mode density limit with ballooning stability at the separatrix Nucl. Fusion. 2018;58:034001.

[11] Myra JR, Russell DA, D'Ippolito DA, Ahn, JW, Maingi R, et al. Reduced model simulations of the scrape-off-layer heat-flux width and comparison with experiment Phys. Plasmas. 2011;18:012305.

[12] Stangeby PC, Canik JM, Whyte DG. The relation between upstream density and temperature widths in the scrape-off layer and the power width in an attached divertor. Nucl. Fusion. 2010;50(12):125003.

[13] Militello F, Naulin V, Nielsen AH. Numerical scalings of the decay lengths in the scrape-off layer. Plasma Phys. Contr. Fusion. 2013;55:074010.

[14] Halpern F D, Ricci P. Velocity shear, turbulent saturation, and steep plasma gradients in the scrape-off layer of inner-wall limited tokamaks Nucl. Fusion. 2017;57:034001.

[15] Nielsen AH, Juul Rasmussen J, Madsen J, Xu GS, Naulin V, Olsen JMB, Løiten M, Hansen SK, Yan N, Tophøj L, Wan BN. Numerical simulations of blobs with ion dynamics Plasma Phys. Contr. Fusion. 2017;59(2):025012.

[16] Dudson BD, Leddy, B. Hermes: global plasma edge fluid turbulence simulations Plasma Phys. Contr. Fusion. 2017;59(5):054010

[17] Halpern FD, Ricci P, Jolliet S, Loizu J, Morales J, Mosetto A, et al. The GBS code for tokamak scrape-off layer simulations J. Comput. Phys. 2016;315:388-408

[18] Tamain P, Bufferand H, Ciraolo G, Colin C, Galassi D, Ghendrih Ph, Schwander F, Serre E. The TOKAM3X code for edge turbulence fluid simulations of tokamak plasmas in versatile magnetic geometries J. Comput. Phys. 2016;321:606-623

[19] Sieglin B, Eich T, Faitsch M, Herrmann A, Scarabosio A, et al. Investigation of scrape-off layer and divertor heat transport in ASDEX Upgrade L-mode Plasma Phys. Contr. Fusion 2016;58(5):055015.

[20] Braginskii SI. Transport processes in a plasma Rev. Plasma Phys. 1965;1:205

[21] Madsen J, Naulin V, Nielsen AH, Juul Rasmussen J. Collisional transport across the magnetic field in drift-fluid models Phys. Plasmas. 2016;23(3):032306.

[22] Fundamenski W, Garcia OE, Naulin V, Pitts RA, Nielsen AH, Juul Rasmussen J, Horacek J, Graves JP et. al. Dissipative processes in interchange driven scrape-off layer turbulence Nucl. Fusion 2007;47(5):417

[23] Fundamenski W. Parallel heat flux limits in the tokamak scrape-off layer Plasma Phys. Control. Fusion. 2005;47:R163.

[24] Zweben SJ, Boedo JA, Grulke O, et. al. Edge turbulence measurements in toroidal fusion devices Plasma Phys. Control. Fusion. 2007;49(7):S1.

[25] Myra JR, D'Ippolito DA, Russel DA. Turbulent transport regimes and the scrape-off layer heat flux width Phys. Plasmas 2015;22:042516.

[26] Riva F, Colin C, Denis J, Easy L, Furno I, Madsen J, et al. Blob dynamics in the TORPEX experiment: a multi-code validation Plasma Phys. Control. Fusion. 2016;58(4):044005

[27] Militello F, Walkden NR, Farley T, Gracias WA, Olsen J, Riva F et al. Multi-code analysis of scrape-off layer filament dynamics in MAST Plasma Phys. Control. Fusion. 2016;58(10):105002. 
Scrape-off layer power fall-off length from turbulence simulations of ASDEX Upgrade L-mode19

[28] Jorge R, Ricci P, Halpern FD, Loureiro NF, Silva C. Plasma turbulence in the scrape-off layer of the ISTTOK tokamak Phys. Plasmas. 2016;23(10):102511. 